\title{
Comparative Evaluation of Tramadol Wound Infiltration over Bupivacaine for Postoperative Analgesia in Children Undergoing Inguinal Herniotomy and Orchidopexy
}

\author{
Abdul Qayoom Lone, M.D., Nazia Nazir, M.D., Shehnaz Gilani, M.D., Mohammad Ommid, M.D., Showkat \\ H. Nengroo, M.D., Akhtar Naqib, M.D., Imtiyaz Naqash, M.D. \\ Department of Anesthesiology \& Critical Care, Sher-i-Kashmir Institute of Medical Sciences, Srinagar, Kashmir (J\&K).
}

\section{A B S T R A C T}

BACKGROUND: Recent clinical studies have demonstrated that children experience a similar severity of post operative pain as adults and that even preterm infants demonstrate alterations in heart rate, blood pressure and oxygen saturation in response to pain.

OBJECTIVE: To study the advantage of tramadol wound infiltration over bupivacaine on post operative pain relief in children following inguinal herniotomy and orchiodopexy

METHODS: In this prospective single blinded randomized controlled study, 705 children aged between 1-7 years undergoing elective repair of unilateral inguinal hernia and orchiodopexy were randomly divided into 3 groups of 25 each. Group A (Tramadol) à wound infiltration with $2 \mathrm{mg} / \mathrm{Kg}$ Tramadol in $0.2 \mathrm{ml} / \mathrm{Kg}$ saline, Group B (Bupivacaine) à wound infiltration with $0.2 \mathrm{ml} / \mathrm{Kg}$ of $0.25 \%$ of Bupivacaine and Group C (Tramadol i/m) à $2 \mathrm{mg} / \mathrm{Kg}$ tramadol intra muscularly 20 minutes before the end of surgery. Children were assessed post-anesthesia for pain score and discharged from Post Anesthesia Care Unit when they achieved a score of 10.

RESULTS: Group A had lower pain scores than group B and C for the first 24 hours in the postoperative period. The other two parameters pulse rate and respiratory rate were also lower in group A. Moreover use of rescue analgesia was significantly lower in groupA.

CONCLUSION: Wound infiltration with tramadol is a good choice for post operative analgesia in children undergoing inguinal herniotomy and orchidopexy. JMS $2011 ; 14(2): 52-55$

Key words: Tramadol, postoperative analgesia, bupivacaine, herniotomy, orchidopexy

International Association for study of Pain defined pain as an "unpleasant sensation \& an emotionally charged experience associated with actual or potential tissue damage or described in terms of such damage. ${ }^{1}$ Recent clinical studies have demonstrated that children experience a similar severity of post operative pain as adults and that even preterm infants demonstrate

Correspondence and reprint requests to:

Dr. Abdul Qayoom Lone

Additional Professor,

Department of Anesthesiology \& Critical Care,

Sher-i-Kashmir Institute of Medical Sciences,

Srinagar, Kashmir (J\&K) India - 190011

Email:drloneaq@hotmail.com alterations in heart rate, blood pressure and oxygen saturation in response to pain. ${ }^{2}$ The subjectivity and multidimensional nature of pain requires clinicians to approach pain assessment using a combination of child's verbal report in conjunction with observation and psychological measures. The optimal methods to achieve analgesia after inguinal hernia repair in children are not clear. Techniques include: administration of opioids, NSAIDs, peripheral nerve blocks, caudal block and wound infiltration with local agents. Studies have shown that instillation of local anesthetics into a wound provides post-operative pain relief following hernia repair which is as effective as that provided by a post-operative caudal block. ${ }^{3}$ Several clinical studies have shown that Tramadol might have peripheral local anaesthetic like 
properties ${ }^{4,5}$ Various observations of behavior techniques are useful for pain assessment in children as Facies Score, Modified Childrens Hospital of Eastern Ontario Pain Scale (mCHEOPS), Visual analogue scale, Observer pain scale etc. In children, anesthetist can assess child's mood on a scale of 1 to 5 in the anesthesia room immediately before induction. ${ }^{6}$ as Mood Grades (Cheerful, Apprehensive \& Cooperative, Tearful and co operative, Uncooperative, Terrified and virtually unmanageable).

\section{Methods}

This prospective single blinded randomized controlled study looking at the advantage of tramadol wound infiltration over bupivacaine on post operative pain relief in children aged 1-7 years following inguinal herniotomy and orchiodopexy included 75 children who were divided randomly into 3 groups of 25 each as: Group A (Tramadol) in whom wound infiltration with $2 \mathrm{mg} / \mathrm{Kg}$ tramadol in $0.2 \mathrm{ml} / \mathrm{Kg}$ saline was done by the surgeon, Group B (Bupivacaine) in whom wound infiltration with $0.2 \mathrm{ml} / \mathrm{Kg}$ of $0.25 \%$ of bupivacaine was done by the surgeon and Group C (Tramadol i/m) $2 \mathrm{mg} / \mathrm{Kg}$ tramadol injected intra muscular 20 minutes before the end of surgery. A standardized anaesthesia technique was carried out in all the patients with mandatory monitoring. Before the end of surgery, local infiltration into the region of surgical incision was performed by the surgeon. Children were reversed, extubated and assessed in Post Anesthesia Care Unit by nurses who were unaware of the group assignment. Pulse rate, respiratory rate and pain score were assessed using FACIES pain score.

Patients with pain score $>4$ received $20 \mathrm{mg} / \mathrm{kg}$ paracetamol rectally. Children were discharged from Post Anesthesia Care Unit when they achieved recovery score of 10 . The frequency of vomiting, pruritus, local allergic reaction and analgesic used were also recorded. The pain scores at 1, 4, 8, 12 and 24 hours were noted. Age, weight, duration of Surgery, duration of GA,1st analgesic requirement and average pain scores were compared with ANOVA (one way analysis of variance). All d were collected and comparison between groups was expressed as arithmetic mean (mean \pm SD) and analyzed using standard statistical tests and inferences were drawn accordingly.

\section{Results}

The mean age (years) of patients was $3.4 \pm 1.4,3.7 \pm 1.1$ and $3.9 \pm 1.4$ in groups $\mathrm{A}, \mathrm{B}, \mathrm{C}$ respectively (Table 1 ). The weights of all children ranged from $8-24 \mathrm{Kg}$, the average weight (mean \pm S.D.) in $\mathrm{Kg}$ was $15.8 \pm 3.1$, 15.2 \pm 2.9 , and 16.3 \pm 3.1 in groups A,B,C respectively, being statistically insignificant on comparison. $(p=0.421)$.

Mean Respiratory Rate (breaths/min); At 1 hour it was 22.0 $\pm 2.8,27.5 \pm 2.5$ and 26.6 \pm 3.1 in groups $A, B$ and C respectively. Intergroup comparison revealed a statistically significant $(p=$ 0.000 ) difference in respiratory rates between groups $\mathrm{A}$ and $\mathrm{B}$ but not significant between groups B and C. Similarly at 4, 8, 12 and 24 hour intervals the respiratory rates recorded were $20.2 \pm 2.0$, $28.3 \pm 4.0$ and $27.5 \pm 2.7$ at 4 hour; $20.5 \pm 2.7,25.8 \pm 3.5$, and 25.3 \pm 3.2 at 8 hour, $19.6 \pm 2.5,26.7 \pm 1.6$ and $24.3 \pm 3.4$ at 12 hour and $19.3 \pm 1.6,26.1 \pm 3.9,27.2 \pm 4.0$ at 24 hour interval in groups $\mathrm{A}, \mathrm{B}$ and $\mathrm{C}$ respectively. The statistical difference was significant $(p=0.000)$ when groups A \& B and A \& C were compared at these intervals but insignificant $(p=0.376,0.596 .0 .012,0.852)$ when compared between Groups B and C at 4, 8, 12 and 24 hour intervals respectively (Fig. 1).

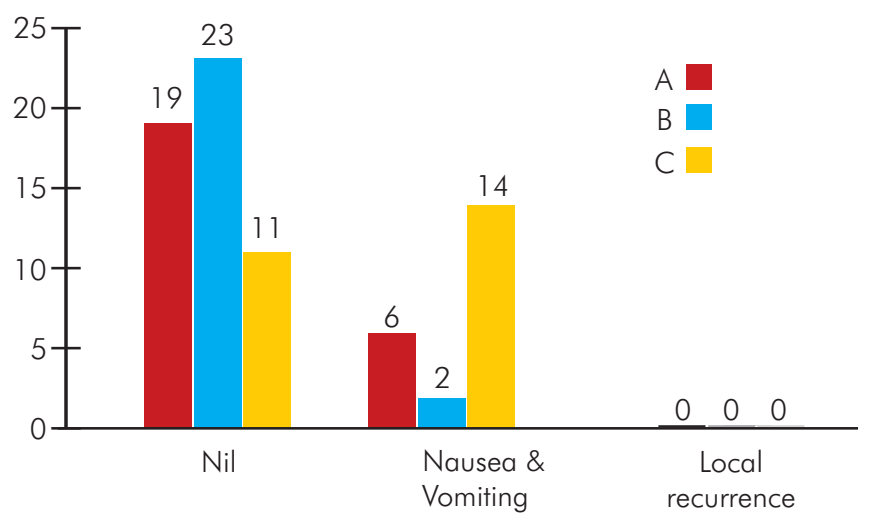

FIGURE 1: Graph showing postoperative complications in the three groups

Mean Pulse Rate: Pulse rate (beats/min.) shared almost a similar trend as respiratory rate at different study stages. At 1 hour postoperatively the rate was at its minimum in group A (97.7 \pm 8.8) compared to group B (110.4 \pm 10.7$)$ and group C (110.8 \pm 11.1), showing a statistically significant $(p=0.000)$ difference between Group A \& B, but non significant between B \& C ( $p$ $=0.912$ ). Similarly the recorded pulse rates were 95.5 \pm 5.0 , 111.6 \pm 11.6 and 111.1 \pm 11.4 at 4 hours; $94.6 \pm 6.2,107.2 \pm 10.3$ and $108.0 \pm 8.6$ at 8 hours: and 92.8 $\pm 5.1,106.2 \pm 9.2$ and $106 \pm 8.6 \mathrm{bpm}$ at 12 hour interval in groups A, B \& C respectively.

TABLE 1. Distribution of Age \& Weight

\begin{tabular}{|c|c|c|c|c|c|c|c|}
\hline & & Max & Min & Mean \pm S.D & \multicolumn{3}{|c|}{ ' $p$ ' value (inter group) } \\
\hline \multirow[t]{3}{*}{ Age (years) } & A & 7 & 1 & $3.4 \pm 1.4$ & $A \& B$ & 0.430 & \multirow{3}{*}{0.364 (not significant) } \\
\hline & B & 6 & 1 & $3.7 \pm 1.1$ & $A \& C$ & 0.158 & \\
\hline & $C$ & 7 & 2 & $3.9 \pm 1.4$ & $B \& C$ & 0.528 & \\
\hline \multirow[t]{3}{*}{ Weight $(\mathrm{kg})$} & A & 22 & 10 & $15.8 \pm 3.1$ & $A \& B$ & 0.453 & \multirow{3}{*}{0.421 (not significant) } \\
\hline & B & 20 & 8 & $15.2 \pm 2.9$ & $A \& C$ & 0.573 & \\
\hline & $C$ & 24 & 11 & $16.3 \pm 3.1$ & $B \& C$ & 0.1 & \\
\hline
\end{tabular}


The statistical difference was significant $(p=0.000)$ when groups $A \& B$ and $A \& C$ were compared at these intervals but insignificant $(p=0.852,0.767 .0 .760$, ) when compared between Groups $\mathrm{B}$ and C.. The pulse rates at 24 hour interval were 91.6 \pm 4.2 , $105.1 \pm 10.2$ and $110.8 \pm 9.2$ showing a significant difference between $\mathrm{A} \& \mathrm{~B}(p=0.000)$ and $\mathrm{A} \& \mathrm{C}(p=0.000)$ and between $\mathrm{B}$ $\& \mathrm{C}(0.021)$.

Pain Score: As shown in figure 2, the pain score at 1 hour postoperatively was minimum in group A $(1.4 \pm 0.3)$ compared to group B $(4.7 \pm 0.3)$ and Group C $(6.1 \pm 0.4)$, the difference being significant between Groups A \& B (0.000), A\& C (0.000), and $\mathrm{B} \& \mathrm{C}(0.005)$. On comparing the pain score at 4 hours, it was lowest in group $\mathrm{A}(2.0 \pm 0.2), \mathrm{B}(5.8 \pm 0.3)$ and $\mathrm{C}(6.6 \pm 0.4)$ being significant $(p=0.000)$ between A \& B and A \& C, but insignificant between $\mathrm{B} \& \mathrm{C} \quad(p=0.061)$, significant difference $(p=0.000)$ between $\mathrm{A} \& \mathrm{~B}$ and $\mathrm{A} \& \mathrm{C}$ and between $\mathrm{A} \& \mathrm{C}(p$ $=0.001$ ) were observed between the groups at 8 hour interval with values of $1.9 \pm 0.2,5.7 \pm 0.3$ and $7.1 \pm 0.3$ in groups $A, B$ and C respectively. Scores at 12 hour were $2.4 \pm 0.3,6.2 \pm 0.3$ and 6.7 \pm 0.3 in $\mathrm{A}, \mathrm{B}$ and $\mathrm{C}$ respectively, being significant $(p=0.000)$ between $\mathrm{A} \& \mathrm{~B}$ and $\mathrm{A} \& \mathrm{C}$ only. Comparing pain score at 24 hour showed lowest values in group A (2.2 \pm 0.3$)$, then group B (6.2 $\pm 0.3)$ and group $\mathrm{C}(7.2 \pm 0.4)$ with significant $(p=0.000)$ difference between $A \& B$ and $A \& C$ Conly.

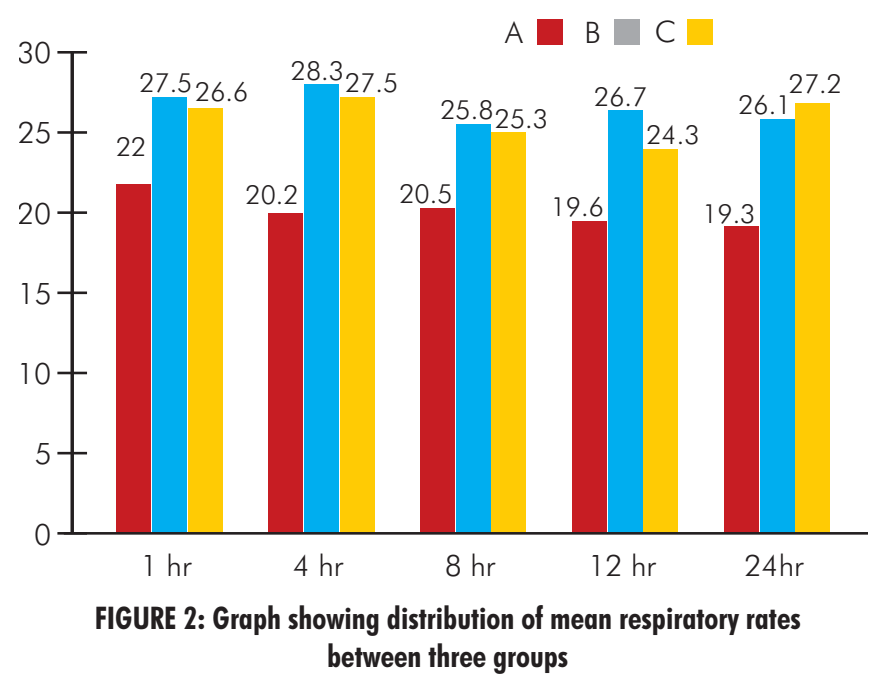

Post operative Complications: 6 (24\%) children in group A, 2 $(8 \%)$ in group B and $14(56 \%)$ children in group C had nausea/vomiting. No child had any local reaction. Comparing the postoperative complications among all 3 groups $A \& B, A \& C$ and B \& C, no significant variation $(p>0.05)$ was observed. Overall $53(70.6 \%)$ children in our study did not develop any postoperative complication. (Fig. 3)

\section{Post operative Analgesia requirement}

In the first hour, inter group comparison revealed statistically significant difference $(p=0.049)$ of analgesic requirement between $A \& C$, but insignificant between groups A \& B and B \& C. Further comparison revealed significant differences in fourth

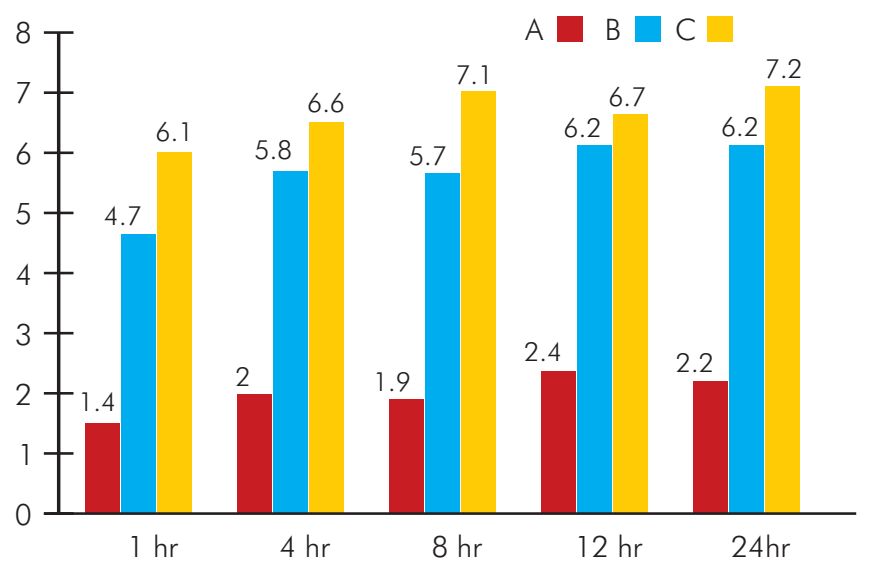

FIGURE 3: Graph showing distribution of mean pain score between the three groups

hour between $\mathrm{A} \& \mathrm{~B}$ (0.001), and A \& $\mathrm{C}$ (0.032): at eight hour between A \& B (0.049), at 12th hour between groups A \& B $(0.049)$ and group B \& C (0.020). At 24 hours postoperatively, none of the children in any of the three groups required any form of rescue analgesic. The overall requirement of rescue analgesia was lowest in Group A. However, the difference was statistically significant at fourth hour $(p=0.003)$ and12 hour $(p=0.005)$.

\section{Discussion}

Acute pain is one of the most common experiences a child will have as a result of injury, illness or medical procedures. The pediatric experience of acute pain involves the interaction of physiological, psychological, behavioral, developmental and situational factors. Recent clinical studies have demonstrated that children experience a similar severity of post operative pain as adults and that even preterm infants demonstrate alterations in heart rate, blood pressure and oxygen saturation in response to pain. ${ }^{2}$

Compared with adults, paediatric surgical patients present some unique problems. Opioid clearance is prolonged and are apparently more prone to opioid induced respiratory depression. Because of developmental, cognitive and emotional differences the assessment of pain in paediatric patients can be more difficult.

Currently available post operative pain management modalities for children are oral preparations, rectal preparations, parenteral preparations \& regional blocks. Studies have shown that instillation of local anesthetic into a wound provides postoperative pain relief following hernia repair which is as effective as that provided by a post-operative caudal block. ${ }^{3}$

The effect of infiltration is short lived but may be increased by the insertion of catheter into or near the wound. ${ }^{12}$ Opioids provide good pain relief particularly in severe pain. However their use is restricted because of potential side effect. Nevertheless the use of local anaesthetic agents may also avoid many of the potential problems associated with opioids. ${ }^{13}$ Various observations of behavior techniques useful for pain assessment in children include: FACIES SCORE - Smiley test (Facial expression of pain score), suitable for pre-school children, where 
child is asked to select from a series of pictures of facial expression ranging from laughter to crying \& choose the one that depicts how he or she feels now. ${ }^{7}$ Others include m-CHEOPS (Modified Childrens Hospital of Eastern Ontario Pain Scale), Visual Analogue Scale, Observer Pain Scale etc.

Tramadol is an atypical centrally acting opioid producing antinociceptive and analgesic effects through spinal and supraspinal sites. However, several clinical studies have shown that it might have peripheral local anaesthetic type properties. ${ }^{4,5}$ Bupivacaine is an amino-amide local anaesthetic used since 1963 for infiltration, peripheral nerve blocks and neuraxial blocks.

The aim of the present study was to compare the post operative analgesic effect of surgical wound infiltration of tramadol $2 \mathrm{mg} / \mathrm{kg}$ body weight with wound infiltration of $0.25 \%$ bupivacaine $0.2 \mathrm{ml} / \mathrm{kg}$ body weight and intra muscular tramadol $2 \mathrm{mg} / \mathrm{kg}$ body weight, in children undergoing inguinal herniotomy and orchidopexy.

The physical characteristics, age and weight were comparable in all the three groups $(p>0.05)$. In the present study, pain scores were evaluated using the FACIES pain score, and respiratory rate as well as the pulse rate were evaluated for the first hour in the post anesthesia care unit and for the next 24 hours in the pediatric surgery ward. At 1,4, and 8 hour time intervals, the comparative parameters of mean pain score, respiratory rate and pulse rate were statistically significant between group $A \& B$ and groups A \& $C(p=0.001)$. Similar results were obtained by Yavuz Demimaran et al study for age group of 1-6 years scheduled for herniotomy comparing tramadol versus bupivacaine wound infiltration.

Intergroup comparison revealed a statistically significant difference $(p=0.000)$ between groups $\mathrm{A} \& \mathrm{~B}$ and $\mathrm{A} \& \mathrm{C}$ at 12 hour, as regards mean pain score, respiratory rate and pulse rate. At 24 hour, mean pain score, respiratory rate and pulse rate was lowest in group A and, the difference was found to be statistically significant between the groups. The overall requirement of rescue analgesia in the form of paracetamol $20 \mathrm{mg} / \mathrm{Kg}$ body weight rectally was lowest in group A. However the difference between the three groups was statistically significant only at the fourth hour $(p=0.003)$ and 12 hours $(p=0.005)$ postoperatively. No significant postoperative complication was found in any of the three groups. Study by Hanife Altunkaya et al. ${ }^{9}$ demonstrated that tramadol has local anaesthetic action and that rescue analgesic need was minimal with the use of tramadol. Gerek et $a^{10}$ in his study showed that subcutaneous tramadol infiltration can provide effective analgesia and may have anti-inflammatory effects. Studies by Kargi et al ${ }^{11}$ and Pang WW et al. ${ }^{14}$ were similar to findings in our study with regards to quality and duration of analgesia by tramadol. The difference between some studies is with regards to local reaction. In our study none of the children had any local reaction at the surgical site. Our study showed that analgesic effect was longest in the tramadol infiltration group, in accordance with a study by Pozos-Guillen Ade Jet al. ${ }^{15}$

In conclusion, postoperative analgesia was significantly prolonged and fewer analgesics were required after wound infiltration with tramadol hence demonstrating that tramadol is a good choice for postoperative analgesia in children undergoing inguinal herniotomy and orchidopexy.

\section{References}

1. IASP - International Association for the study of pain, pain terms: A list with definitions and notes on usage. Pain 1979; 6:249-52.

2. Joseph D Tobias, et al. Advances in pain Management in children. ASANews letter, August 2000 volume 64.

3. Machotta A et al. Comparison between instillation of bupivacaine versus caudal analgesia for post-operative analgesia following inguinal herniotomy in children. Peadiatric Anesthesia 2003;13:397-402.

4. Tsai YC, Chang PJ et al. Direct tramadol application on sciatic nerve inhibits spinal somatosensory evoked potentials in rats. Anaesthesia Analgesia 2001;92:1547-51.

5. John B. Cose, et al. Oral tramadol for the treatment of pain of 7-30 days Duration in children. Anesthesia Analgesia 2003;96:78-81.

6. Reid, MF et al. Day-case herniotomy in children: A comparison of ilio-inguinal nerve block and wound infiltration for post-operative analgesia. Anesthesia 1987; 42: 658-61.

7. Beyer J, Aradine C. Content validity of an instrument to measure young childrens' perception of the intensity of their pain.Journal of Pediatric Nursing 1986;1:386-95.

8. Yavuz Demiraran et al. Does tramadol wound infiltration offer an advantage over bupivacaine for postoperative analgesia in children following herniotomy. Paediatric Anaesthesia 2006;16:1047-50.

9. Hanife Altunkaya, et al. The post operative Analgesic effect of tramadol when used as subcutaneous local Anesthetic. Anesth Analgesia 2004;96:1461-64.

10. Gercek A, Eti Z, Gogus FY, Sav A, et al. The analgesic and anti-inflammatory effects of subcutaneous bupivacaine, morphine \& tramadol in rats. Agri 2004;16(3):53-58.

11. Kargi E, et al. Tramadol as a local anesthetic in tendon repair surgery of the hand. IntMed Res 2005; 36 (5):971-75.

12. Catherine $\mathrm{E}$ Wood et al. Complication of continous epidural infusions for postoperative analgesia in children. CanJ Anaesthesia 1994;41:613-20.

13. Chung $\mathrm{F}$ et al. Postoperative pain in ambulatory surgery Anaesthesia Analgesia 1997;85:806-16.

14. Pang WW, Mok MS, et al. Intradermal injection of tramadol has local anesthetic effects: a comparison with lidocaine. Acta Anaesthesiology Sin 1998;36(3):133-36.

15. Pozos-Guillen Ade J, et al. Analgesic efficacy of tramadol by route of administration in a clinical model of pain. Proc West pharmacol 2005;48:61-64. 